\title{
Cambios climáticos en las sierras de Córdoba (Argentina) DuRANTE El holoceno. Aportes a las reconstrucciones CLIMÁTICAS A TRAVÉS DEL ANÁLISIS DE SILICOFITOLITOS DEL SITIO ARQUEOLÓgICO El Alto 3
}

\author{
MELISA A. GIORGIS ${ }^{1 *}$, MARIA L. LOPEZ², DIEGO RIVERO ${ }^{3}$ y ANA M. CINGOLANI ${ }^{1}$
}

\begin{abstract}
Summary: Holocene paleoclimatic variation in The Cordoba Mountains (Argentina). A contribution to climate reconstruction though silicophytolith analysis from archaeological site El Alto 3 . The analysis of silicophytoliths is an increasingly used tool for paleoclimatic reconstructions. However, in Córdoba climatic reconstruction has been performed with other techniques. In this work we aimed to calculate international climatic indices through silicophytoliths, validate these indices with the current vegetation and climate distribution in the Mountains, and present the first paleoclimatic reconstruction through silicophytoliths for the oldest archaeological site in Cordoba. The international indices were consistent with the patterns of the current vegetation and climate distribution; though the coolness index was more robust and sensitive than the aridity index. Along the analyzed profile the silicophytoliths indicated a cold and humid/wet climate, which is characteristic of a mountain site, but they showed important variation in the coolness and aridity index. The climatic reconstruction was consistent with the previous studies, since it showed a peak of aridity at the beginning of the Holocene, followed by a wetter and warmer climate than the current climate, a subsequent peak of higher aridity and warm was coincident with the presence of the Arecaceae family, and finally it showed a temperature reduction and precipitation increase until the present.
\end{abstract}

Key words: Mountains, climate change, $\mathrm{C}_{3}$ and $\mathrm{C}_{4}$, vegetation change.

Resumen: El análisis de silicofitolitos es una herramienta cada vez más utilizada para la reconstrucción paleoclimática. Sin embargo, en Córdoba estas reconstrucciones se han realizado con otras técnicas. En este trabajo nos propusimos calcular los índices climáticos internacionales a partir de los silicofitolitos, validar dichos índices utilizando la distribución de la vegetación y clima actual de las sierras, y presentar la primera reconstrucción paleoclimática a través de silicofitolitos para el sitio arqueológico más antiguo de Córdoba. Los índices internacionales fueron consistentes con los patrones de distribución de la vegetación y clima actual, aunque el índice de frío fue más confiable y sensible que el índice de aridez. A lo largo de perfil analizado los datos de silicofitolitos indicaron un clima frío y húmedo característico del un sitio de montaña, pero mostraron importantes variaciones en los índices de frío y aridez. La reconstrucción climática fue consistente con los trabajos previos, al mostrar un pico de aridez al comienzo del Holoceno, seguido por un clima más húmedo y cálido que el actual, un posterior pico de mayor aridez y calor coincidente con la presencia de la familia Arecaceae y finalmente marca una disminución en la temperatura y aumento de las precipitaciones hasta la actualidad.

Palabras clave: Montañas, cambio climático, $\mathrm{C}_{3}$ y $\mathrm{C}_{4}$, cambios en la vegetación.

1 Instituto Multidisciplinario de Biología Vegetal, CONICET- FCEFyN, Universidad Nacional de Córdoba, Córdoba, Argentina.

2 División Arqueología, Facultad de Ciencias Naturales y Museo. Universidad Nacional de La Plata. CONICET.

${ }^{3}$ Centro de Estudios Históricos "Carlos Segreti”. CONICET.

*melisagiorgis@hotmail.com 


\section{INTRODUCCIÓN}

Las reconstrucciones paleoclimáticas han permitido entender cómo las variaciones climáticas ocurridas en los últimos milenios han modulado los patrones de distribución de los ecosistemas y biomas, e influido en la distribución y expansión de la especie humana (Lomolino et al., 2010). Estos conocimientos son clave no sólo para entender los patrones de distribución de los biomas actuales y pasados, sino también para predecir su futuro y planificar adecuadas estrategias de manejo y conservación.

El análisis de silicofitolitos en perfiles de suelo ha sido de gran ayuda para la reconstrucción climática y florística en muchos lugares del mundo (por ejemplo: Fredlund \& Tieszen, 1994; Bremond et al., 2008). Principalmente las gramíneas (familia Poaceae) forman fitolitos de sílice amorfo (silicofitolitos), cuyas formas generales caracterizan a cada una de las principales subfamilias (Pooideae, Chloroideae, y Panicoideae). Los silicofitolitos de las Pooideae son generalmente circulares, rectangulares, elípticos, crestados o con formas oblongas, ocurren en pastos principalmente $\mathrm{C}_{3}$, que están concentrados a altas altitudes y latitudes. Mientras que los silicofitolitos de las Chloroideae tienen forma de silla de montar (saddle) corresponden generalmente a especies $\mathrm{C}_{4}$ característicos de lugares cálidos, áridos y semiáridos. Finalmente los fitolitos de las Panicoideas incluyen formas que varían entre cruces y bilobados-halteriformes (crosses and dumbbells), y también corresponden generalmente a pastos $\mathrm{C}_{4}$ predominantes en lugares calurosos de regiones tropicales y subtropicales (Twiss, 1992). La asociación fitolítica fósil puede ser recuperada de los suelos y de distintos tipos de sedimentos y ser usada en la reconstrucción de la composición de la vegetación y del clima (Rovner, 1971; Fredlund \& Tieszen, 1997). La reconstrucción climática basada en la utilización de silicofitolitos es reconocida en todo el mundo (Twiss, 1992; Boyd, 2005; del Puerto et al., 2006). Para ello se calculan índices globales de precipitación y de temperatura utilizando la información de la composición de las tres subfamilias. Sin embargo, si bien los patrones de distribución de las gramíneas son claros tanto a nivel global (Mooney \& Ehleringer, 1997), como regional (Cabido et al., 2008), estos patrones pueden verse alterados localmente por razones evolutivas particulares y factores relacionados con el régimen actual e histórico de disturbio del sistema (Cingolani et al., 2008). De esta manera se hace necesario evaluar a nivel local cómo los factores climáticos se relacionan con los índices climáticos globales obtenidos de la proporción de subfamilias. Esto es particularmente importante en América del Sur, donde el estudio de los silicofitolitos en muchas regiones es más reciente que en otros continentes (Zucol et al., 2008).

En Argentina los análisis de silicofitolitos se han utilizado para la reconstrucción climática, paleobotánica y arqueobotánica. Por ejemplo en las regiones costeras, del litoral y Patagonia los análisis de silicofitolitos en paleosuelos y suelos han dado excelentes resultados en la caracterización y determinación de la vegetación y clima pasados (Andreis, 1972; Zucol \& Brea, 2000, 2001; Zucol et al., 2001, 2002, 2003, 2004; Osterrieth et al., 2002, 2008, 2009; Fernández Honaine et al., 2006, 2009a,b; Borrelli et al., 2008, 2011; Osterrieth, 2006; Bonomo et al., 2009). Por otro lado, en el noroeste argentino, los estudios de silicofitolitos se han utilizado en sitios arqueológicos para caracterizar cultivos prehispánicos y la vegetación natural circundante a las áreas cultivadas (Korstanje, 2005, 2009; Korstanje \& Cuenya, 2008, 2010).

En la región central de Argentina existen dos estudios publicados sobre la identificación de silicofitolitos de gramíneas nativas de la provincia de La Pampa (Gallego \& Distel, 2004; Gallego et al., 2004), y un estudio sedimentológico de silicofitolitos en la provincia de San Luis, donde se reconstruyen las condiciones climáticas del Pleistoceno tardío-Holoceno (Strasser et al., 2006). En Córdoba, los trabajos basados en análisis de silicofitolitos han estado orientados principalmente a conocer los recursos domésticos consumidos por el ser humano desde el Holoceno Medio (López, 2007; Medina et al., 2009; Rivero \& López, 2010, 2011). Sin embargo, todavía no se han utilizado para reconstruir las condiciones climáticas del pasado.

En general, la investigación sobre los paleoambientes en la provincia de Córdoba ha sido encarada por medio del análisis de la composición sedimentaria y de la geomorfología del paisaje (Carignano, 1999; Cioccale, 1999; Piovano et al., 2009; Sanabria et al., 2009; Kröhling \& Carignano, 2014) y recientemente también través 
de estudios isotópicos en el perfil del suelo que determinan la proporción de especies $\mathrm{C}_{3} / \mathrm{C}_{4}$ (Silva et al., 2011). Estos estudios han echado luz sobre las condiciones climáticas desde finales del Pleistoceno, esclareciendo el marco ambiental donde se desarrolló la vida humana desde que los cazadores-recolectores habitaban la región hasta la actualidad. En general, señalan un período cálido y húmedo hasta aproximadamente la mitad del Holoceno, con un posterior desecamiento del clima hasta alcanzar un pico de mayor aridez y calor entre los 4000 y 1000 años AP (dependiendo del autor y del trabajo) (Carignano, 1999; Piovano et al., 2009; Silva et al., 2011; Kröhling \& Carignano, 2014). Posteriormente a este pico, los estudios citados detectan un progresivo aumento de la humedad y disminución de la temperatura hasta nuestros días, con algunas anomalías como la Anomalía Climática Medieval (o MCA, Medieval Climatic Anomaly por sus siglas en ingles), y posteriormente por la pequeña edad de hielo (o LIA, Little Ice Age por sus siglas en ingles) que concluyó a comienzos del siglo XIX (Piovano et al., 2009; Kröhling \& Carignano, 2014).

Debido a la gran importancia que tiene el conocimiento sobre los cambios climáticos ocurridos durante el Holoceno (Lomolino et al., 2010), en este trabajo nos propusimos brindar nuevos datos sobre los cambios climáticos ocurridos a nivel local en las sierras de Córdoba (centro de Argentina) desde fines del Pleistoceno, a partir del análisis de silicofitolitos. Específicamente, nuestros objetivos fueron: (1) calcular los índices climáticos internacionales a partir de los silicofitolitos encontrados en distintas profundidades del perfil de un sitio arqueológico de las sierras, (2) obtener información de la proporción relativa actual de las tres subfamilias de Poaceae a lo largo de todas las sierras de Córdoba y validar los índices climáticos internacionales relacionando dichos datos con el clima actual de las sierras, y (3) presentar la primera reconstrucción paleoclimática a través de silicofitolitos para la provincia de Córdoba.

\section{Métodos}

\section{Área de estudio}

Las sierras de Córdoba, junto con las sierras de San Luis, forman parte del complejo más austral de las Sierras Pampeanas que se conoce como Sierras Centrales. Están formadas por una serie de cordones montañosos que se extienden más o menos paralelos y orientados con rumbo general norte-sur ocupando un rango de más de $600 \mathrm{~km}$ entre los $29^{\circ} 30^{\prime}$ y $33^{\circ} 30^{\prime}$ de latitud Sur y $63^{\circ} 40^{\prime}$ y $65^{\circ} 20^{\prime}$ de longitud oeste (Fig. 1).

Climáticamente las sierras de Córdoba están divididas según Capitanelli (1979) en dos dominios. Por un lado, las Sierras Grandes y las Sierras Chicas presentan un clima semi-húmedo, con tendencia al semi-seco de montaña. Aproximadamente el 85 $\%$ de las precipitaciones ocurren entre octubre y marzo, con un promedio anual de $725 \mathrm{~mm}$ para Ascochinga (740 m s.n.m.), localidad utilizada para

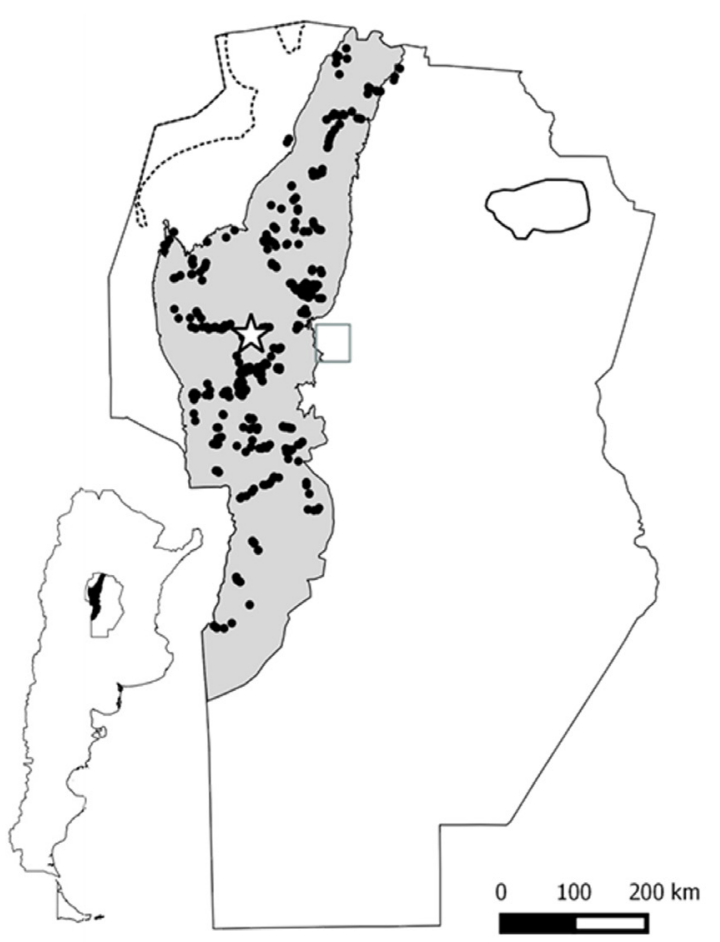

Fig. 1. Ubicación de los sitios de estudio en la provincia de Córdoba (Argentina). La estrella representa la ubicación del sitio arqueológico El Alto 3. Los puntos negros corresponden a los 657 sitios utilizados para la reconstrucción climática actual. El área gris representa la zona serrana de la provincia. Las líneas punteadas delimitan las Salinas Grandes y la línea negra la laguna Mar Chiquita. El cuadrado en el centro del mapa representa la ciudad de Córdoba. 
describir este dominio (Capitanelli, 1979). Para esta misma localidad, la temperatura media anual es de $14,8^{\circ} \mathrm{C}$ (Hijmans et al., 2005). Las Sierras del Norte y de Pocho y Guasapampa, en cambio, pertenecen al dominio semi-desértico de las planicies del Noroeste, sin un invierno térmico marcado. En localidad de Quilino, característica de este dominio, el promedio anual de precipitaciones es de $474 \mathrm{~mm}$, también concentradas en los meses de verano, y la temperatura media anual es de $18,2{ }^{\circ} \mathrm{C}$ (Capitanelli, 1979; Hijmans et al., 2005). La temperatura de las sierras varía también con la altitud siendo el promedio anual a $490 \mathrm{~m}$ s.n.m. de $17,5^{\circ} \mathrm{C}$ en la localidad de Córdoba Capital (Hijmans et al., 2005), adyacente a las sierras, mientras que a 2.700 m s.n.m, en la parte más alta de las sierras, es alrededor de $7.4^{\circ} \mathrm{C}$ (Hijmans et al., 2005; Marcora et al., 2008).

El sitio de estudio se ubica en la ladera este de las Sierras Grandes, donde se extrajo sedimento asociado al sitio arqueológico "El Alto 3", que representa el asentamiento más antiguo de la región central de las Sierras de Argentina (Rivero, 2009) (Fig. 1). El sitio se localiza en el sector nororiental de dicho cordón, a $1650 \mathrm{~m}$ s.n.m. ( $31^{\circ} 23^{\prime} 56^{\prime \prime} \mathrm{S}$ y $64^{\circ} 44^{\prime} 20^{\prime}$ 'W). La zona tiene abundantes afloramientos rocosos y la vegetación está dominada por diferentes especies de gramíneas y algunos arbustos. Puntualmente en el sitio de estudio, la temperatura y la precipitación media anual son de $13,2{ }^{\circ} \mathrm{C}$ y $812 \mathrm{~mm}$, respectivamente (Hijmans et al., 2005; Hoyos, 2012).

El Alto 3 es una de las principales localidades arqueológicas de las Sierras de Córdoba. Su importancia radica en que es el único sitio, hasta el momento, que contiene evidencias de la ocupación humana del sector serrano desde la transición Pleistoceno-Holoceno hasta el Holoceno Tardío (Rivero \& Roldán, 2005). Consiste en un alero de grandes dimensiones con unos $23 \mathrm{~m}$ de largo y una profundidad de $5 \mathrm{~m}$, que se abre en un frente granítico expuesto hacia el sur ubicado en las cotas superiores de la cabecera de una quebrada muy disectada. El alero posee 63 instrumentos de molienda (morteros y molinos planos) excavados en la roca base en el interior del mismo (24) y en una explanada exterior (39) (Rivero, 2009). Durante la excavación en la explanada exterior, que alcanzó $160 \mathrm{~cm}$ de profundidad, se identificaron cuatro componentes arqueológicos, los tres inferiores correspondientes a cazadores-recolectores y el superior a comunidades agro-alfareras (Rivero, 2007). Los sedimentos del sitio son de origen aluvial, con presencia de clastos angulosos, que indicarían una procedencia muy local. Todo el perfil está compuesto por sedimentos arenosolimosos con presencia de fragmentos líticos que van cambiando de forma y tamaño a lo largo del perfil (Rivero, 2009).

Nuestro estudio se concentró en los $120 \mathrm{~cm}$ superiores, donde se obtuvieron seis fechados radiocarbónicos (Rivero y Roldan, 2005; Rivero, 2009) provenientes de los siguientes niveles: 119 $\mathrm{cm}=9790 \pm 80 \mathrm{AP}(\mathrm{LP}-1420), 92 \mathrm{~cm}=7108 \pm 74$ AP (AA68145), $65 \mathrm{~cm}=2990 \pm 70$ AP $($ LP-1502), $58 \mathrm{~cm}=2770 \pm 80$ AP (LP-1287), $42 \mathrm{~cm}=1690$ $\pm 70 \mathrm{AP}(\mathrm{LP}-1604)$ y $22 \mathrm{~cm}=670 \pm 50 \mathrm{AP}(\mathrm{LP}-$ 1278), que corresponderían a los siguiente fechados calibrados, según el programa CalPal (Weninger et al., 2007): $119 \mathrm{~cm}=11210 \pm 50 \mathrm{cal} \mathrm{AP,} 92 \mathrm{~cm}=$ $7930 \pm 70 \mathrm{cal} \mathrm{AP}, 65 \mathrm{~cm}=3180 \pm 110 \mathrm{cal} \mathrm{AP}, 58$ $\mathrm{cm}=2900 \pm 90 \mathrm{cal} \mathrm{AP}, 42 \mathrm{~cm}=1620 \pm 80 \mathrm{cal}$ AP y $22 \mathrm{~cm}=630 \pm 50 \mathrm{cal}$ AP.

\section{Obtención de silicofitolitos y cálculo de índices internacionales}

Las muestras sedimentarias se tomaron del perfil de excavación, que dividimos en 11 niveles artificiales de profundidad, cada $10 \mathrm{~cm}$. En cada nivel tomamos una muestra, por lo cual la más superficial de ellas fue tomada entre 0 y $10 \mathrm{~cm}$, mientras que la más profunda se obtuvo entre 100 y $110 \mathrm{~cm}$. No tomamos muestras por debajo de esta profundidad debido a posibles contaminaciones. De cada una de las 11 muestras separamos una alícuota de 10 gramos para su procesamiento. Las alícuotas fueron tratadas con agua destilada y detergente de laboratorio no iónico (5\%) por 48 horas para lograr la defloculación y desagregación de las partículas. Posteriormente, realizamos el lavado con agua destilada y procedimos a la separación granulométrica con tamiz $\mathrm{n}^{\circ} 270$ (malla de $53 \mu \mathrm{m}$ de luz) y por densidades utilizando la Ley de Stokes, obteniendo de esta manera la fracción entre 7 y $53 \mu \mathrm{m}$ para su observación bajo microscopio. Las alícuotas fueron montadas en aceite de inmersión para su análisis tridimensional bajo microscopio óptico Leica a 400x.

Contabilizamos 400 silicofitolitos derivados de células cortas en cada muestra. Los silicofitolitos 
fueron descritos e identificados siguiendo a Bertoldi de Pomar, (1971, 1975); ICPN-Madella et al., (2005); Pearsall, (1989); Piperno, (2006); Twiss, (1992); Twiss et al., (1969) y Zucol, (1996, 1998, 1999, 2000, 2001) Fredlund \& Tieszen, (1997); Gallego \& Distel, (2004) y Fernández Honaine et al., (2006). Se clasificaron a nivel de las tres subfamilias consideradas indicadoras climáticas (Pooideae, Panicoideae y Chloroideae) (Twiss, 1992). Los silicofitolitos tipo Stipa (Zucol, 1996, 1998, 1999, 2000, 2001; Fredlund \& Tieszen, 1997; Gallego \& Distel, 2004; Fernández Honaine et al., 2006), fueron considerados dentro de la subfamilia Pooideae siguiendo los trabajos de Soreng et al. (2012, 2015). Además distinguimos silicofitolitos globulares con superficie espinosa o globulitas como indicadores de la familia Arecaceae (Bertoldi de Pomar, 1971, 1975) (Apéndice 1).

Para todas las muestras, a partir de la abundancia relativa de silicofitolitos de cada una de las tres subfamilias indicadoras (Pooideae, Panicoideae y Chloroideae) calculamos dos índices climáticos internacionales, uno para temperatura, y otro para humedad (Twiss, 1992). Dado que los valores altos del índice de temperatura señalan climas más fríos, y los valores altos del índice de humedad señalan climas más secos, de aquí en más se denominarán "Índice de frío" e "Índice de aridez", respectivamente.

Índice de frío $=($ Pooideae $\times 100) /($ Pooideae + Panicoideae + Chloroideae)

Los valores altos mayores a 50 dan cuenta de un clima frío de altas latitudes o altitudes, mientras que los valores bajos menores a 20 sugieren temperaturas cálidas típicas de bajas latitudes o altitudes (Twiss, 1992; del Puerto et al., 2006).

Índice de aridez $=($ Chloroideae $\times 100) /$ (Chloroideae + Panicoideae)

Este índice da cuenta de un clima árido si tiene un valor mayor a 40 , mientras que si los valores son menores de 20 el clima es húmedo (Twiss, 1992; del Puerto et al., 2006).

Validación de los indices internacionales utilizando la vegetación y el clima actual

Para analizar si estos índices internacionales se asocian a la precipitación y la temperatura en las sierras de Córdoba, utilizamos datos actuales de vegetación y clima. Tomamos 657 sitios con censos florísticos de plantas vasculares publicados por Cingolani et al., (2010) y Giorgis et al., (2011). Estos censos consisten en la lista de todas las especies vasculares presentes en un área de no más de $400 \mathrm{~m}^{2}$, y una estimación de su cobertura (\%). Estos sitios se ubican en los tres cordones montañosos de Córdoba desde los 367 hasta los $2300 \mathrm{~m}$ de altitud, abarcando toda la extensión de las sierras dentro de la Provincia (Fig. 1). Para cada sitio calculamos la proporción de las tres subfamilias de gramíneas cuyos silicofitolitos son indicadores climáticos (Pooideae, Panicoideae, Chloroideae). Para ello sumamos las coberturas de las distintas especies pertenecientes a cada subfamilia, siguiendo la clasificación de Soreng et al., (2012, 2015), y luego dividimos ese valor por la suma total.

Por otro lado, a partir de mapas de temperaturas y precipitaciones para el período 1950-2000 y 1930-1970 respectivamente (Hijmans et al., 2005; Hoyos, 2012) obtuvimos la temperatura media y precipitación de cada sitio. Posteriormente, para tener una distribución equitativa de datos en los distintos intervalos de precipitación y temperatura, y para disminuir la varianza debido a características puntuales particulares, clasificamos los sitios en intervalos de temperatura cada $1{ }^{\circ} \mathrm{C}$ (en total 9 intervalos, a partir de una variación entre 10 y $19^{\circ} \mathrm{C}$ ) y de precipitaciones cada $100 \mathrm{~mm}$ (en total 6 intervalos, a partir de una variación entre 500 y $1100 \mathrm{~mm}$ ). Para cada categoría resultante de la combinación de intervalos calculamos los valores promedio de: temperatura, precipitaciones y proporción de las tres diferentes subfamilias, utilizando todos los sitios que quedaban incluidos dentro de la categoría. De este modo, obtuvimos un total de 32 puntos que denominaremos "clases climáticas", correspondientes a las distintas combinaciones actuales de precipitación y temperatura. De las 54 combinaciones posibles resultantes de multiplicar 6 franjas de precipitación x 9 franjas de temperatura, sólo 32 existen realmente en las sierras (ver Fig. 3).

Para cada una de las 32 clases climáticas, calculamos los dos índices climáticos (Índice de frío e Índice de aridez) sobre la base de las proporciones promedio de las tres subfamilias 

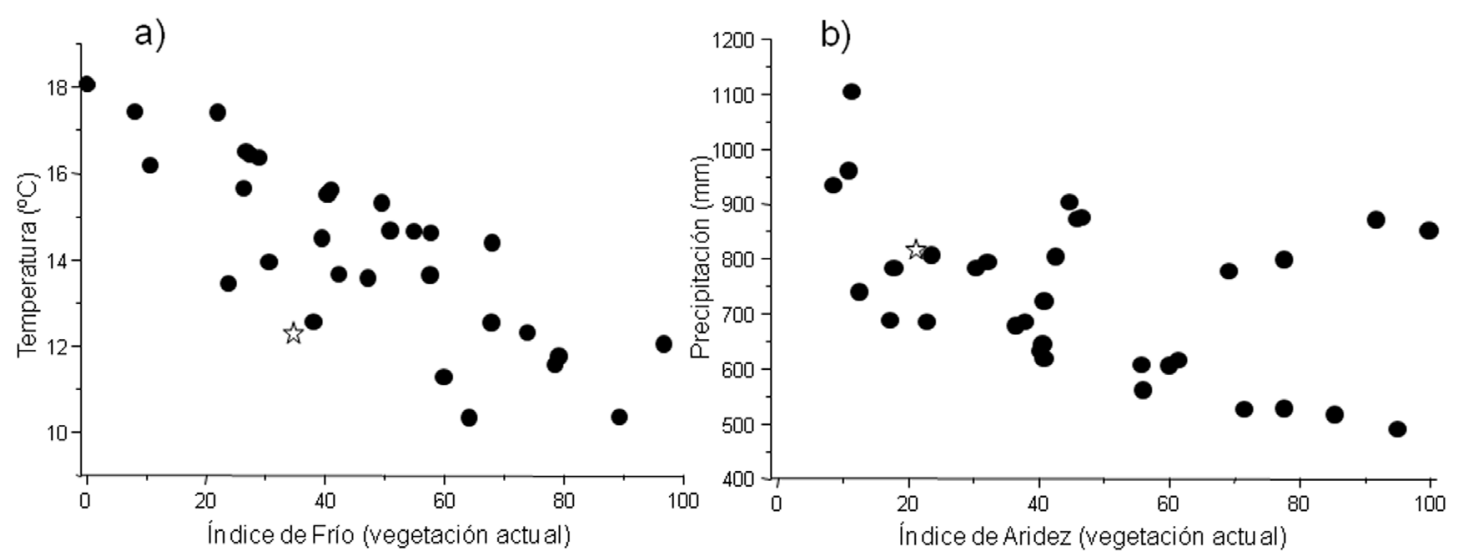

Fig. 2. Relación entre los índices climáticos basados en la vegetación actual y los valores reales de temperatura y precipitación, para las 32 clases climáticas (puntos negros). A modo de referencia, en ambos gráficos se indica con una estrella el punto correspondiente al sitio arqueológico El Alto 3 (cuya vegetación se obtuvo del sitio censado más cercano, a $1 \mathrm{~km}$ de distancia). a) Índice de frío vs temperatura, b) índice de aridez vs precipitación.

presentes. Posteriormente hicimos correlaciones de Pearson entre estos dos índices y los valores de temperatura y precipitación media de cada clase climática $(\mathrm{N}=32)$. Si bien en términos absolutos los índices no reflejan lo mismo que cuando son calculados a partir de silicofitolitos del suelo, esperamos que en términos relativos reflejen las variaciones en temperatura y humedad en las sierras de Córdoba. Es decir, si existe una correlación significativa entre los índices y las variables climáticas respectivas, asumimos que los índices pueden ser aplicados a las Sierras de Córdoba, y que son más robustos cuanto más alta es dicha correlación. Por otro lado, dado que la familia Arecaceae se puede distinguir a nivel de silicofitolitos, analizamos de modo gráfico la relación entre la presencia de esta familia en la actualidad y la precipitación y temperatura.

\section{Reconstrucción paleoclimática a través de silicofitolitos}

Finalmente, sobre la base de los resultados obtenidos, interpretamos los cambios climáticos en el sitio de estudio a lo largo del tiempo. Para ello, graficamos el índice de frío y aridez en función del tiempo. Para la interpretación de los resultados, consideramos que la primera profundidad analizada entre 0 y $10 \mathrm{~cm}$, es aproximadamente representativa de la vegetación y clima actual.

\section{Resultados}

Obtención de silicofitolitos y cálculos de indices internacionales

Dentro de los morfotipos de la subfamilia Panicoideae hemos identificado a Bilobadoshalteriformes (Dumbbell-bilobate), Polilobadoshalteriformes (Dumbbell-polylobate) y Mitad bilobados halteriformes (Half-dumbbell) (Tabla 1). Asimismo, identificamos elementos en forma de estróbilo de base cóncava y ápice sin ornamentación (Rondel), Prismáticos cuadrangulares/rentangulares (Square/rectangle), Circulares/ovalados (Circular/ ovate) y Cuneiformes (Cuneiform), así como el morfotipo Halterio tipo-Stipa (Stipa-type) para la subfamilia Pooideae. Por último, el morfotipo de Silla de montar (Saddle) indicó la presencia de la subfamilia Chloroideae. Además, se identifico la presencia del morfotipo Globular de superficie espinosa (Sphera echinate) en una mínima proporción, indicando la presencia de Arecaceae (Tabla 1).

Se destaca la predominancia de la subfamilia Pooideae a lo largo de todo el perfil del suelo, con algunas variaciones. Estas variaciones en la abundancia de las tres subfamilias determinan variaciones en los índices de frío y aridez (Tabla 1). El índice de frío mostró valores característicos de climas fríos con un mínimo de 68,2 y un máximo de 82,2. Por su parte, el índice de aridez mostró valores muy bajos de 3 a 20,3, 
Tabla 1. Frecuencia absoluta/número de silicofitolitos derivados de células cortas de los distintos morfotipos contados en cada uno de los 11 niveles de profundidad del suelo, de $10 \mathrm{~cm}$ de espesor cada uno, desde la superficie $(\mathrm{N} 1=0-10 \mathrm{~cm})$ hasta el nivel más profundo $(\mathrm{N} 11=100-110 \mathrm{~cm})$. Se indica la suma total clasificada como proveniente de cada una de las tres subfamilias, así como de la familia Arecaceae.

\begin{tabular}{|c|c|c|c|c|c|c|c|c|c|c|c|}
\hline & N1 & N2 & N3 & N4 & N5 & N6 & N7 & N8 & N9 & N10 & N11 \\
\hline Bilobados-halteriformes ${ }^{1}$ & 39 & 49 & 50 & 41 & 50 & 35 & 32 & 23 & 23 & 34 & 27 \\
\hline Polilobados-halteriformes ${ }^{2}$ & 48 & 35 & 47 & 49 & 45 & 39 & 35 & 67 & 49 & 20 & 33 \\
\hline Mitad bilobados halteriformes ${ }^{3}$ & 0 & 0 & 0 & 4 & 10 & 10 & 16 & 13 & 8 & 11 & 5 \\
\hline Total Panicoideas & 87 & 84 & 97 & 94 & 105 & 84 & 83 & 103 & 80 & 65 & 65 \\
\hline Estróbilo de base cóncava y ápice sin ornamentación ${ }^{4}$ & 236 & 207 & 180 & 184 & 177 & 167 & 101 & 108 & 90 & 96 & 170 \\
\hline Halterio Tipo Stipa ${ }^{5}$ & 16 & 20 & 12 & 7 & 5 & 4 & 3 & 12 & 7 & 6 & 11 \\
\hline Cuneiformes $^{6}$ & 5 & 13 & 16 & 21 & 31 & 51 & 38 & 24 & 26 & 23 & 41 \\
\hline Prisma cuadrangular/rentangular ${ }^{7}$ & 40 & 48 & 52 & 51 & 45 & 60 & 94 & 67 & 105 & 85 & 60 \\
\hline Circular/ovalado ${ }^{8}$ & 9 & 14 & 26 & 18 & 15 & 14 & 74 & 75 & 88 & 113 & 32 \\
\hline Esfera escrobiculada ${ }^{9}$ & 0 & 0 & 0 & 0 & 0 & 7 & 0 & 7 & 0 & 1 & 19 \\
\hline Total Pooideas & 306 & 302 & 286 & 281 & 273 & 303 & 310 & 293 & 316 & 324 & 333 \\
\hline Silla de montar ${ }^{10}$ & 7 & 14 & 12 & 24 & 21 & 13 & 7 & 4 & 4 & 11 & 2 \\
\hline Total Chloroideas & 7 & 14 & 12 & 24 & 21 & 13 & 7 & 4 & 4 & 11 & 2 \\
\hline Globular de superficie espinosa ${ }^{11}$ & 0 & 0 & 5 & 1 & 1 & 0 & 0 & 0 & 0 & 0 & 0 \\
\hline Total Arecaceae & 0 & 0 & 5 & 1 & 1 & 0 & 0 & 0 & 0 & 0 & 0 \\
\hline Total cel. Cortas & 400 & 400 & 400 & 400 & 400 & 400 & 400 & 400 & 400 & 400 & 400 \\
\hline Índice de Frío & 76,5 & 75,5 & 71,5 & 70,2 & 68,2 & 75,7 & 77,5 & 73,2 & 79 & 81 & 83,2 \\
\hline Índice de Aridez & 7,4 & 14,3 & 11 & 20,3 & 16,7 & 13,4 & 7,8 & 3,7 & 4,8 & 14,5 & 3 \\
\hline
\end{tabular}

1: Dumbbell-bilobate; 2: Dumbbell-polylobate; 3: Half-dumbbell; 4: Rondel; 5: Stipa-type; 6: Cuneiform; 7 : Square/rectangle; 8: circular/ovate; 9: Sphera scrobic.; 10: Saddle; 11: Sphera echinate.

característicos de climas húmedos a sub-húmedos.

Validación de los índices internacionales utilizando la vegetación y el clima actual

Tal como esperábamos, el índice de frío calculado a partir de la distribución actual de las subfamilias se correlacionó estrechamente con la temperatura actual $(r=-0,84, P<0,001, N=32$, Fig. 2a). También se correlacionó aunque más débilmente, con la precipitación $(r=0,44, P=0,013, N=32)$, señalando que las clases climáticas con una vegetación indicadora de frío, también tienden a ser más húmedas (lo cual también se refleja en la correlación entre la temperatura media y la precipitación media, $r=-0.56$, $P=0,001, N=32$, ver Fig. 3). Por otro lado, el índice de aridez se correlacionó negativa y significativamente con la precipitación ( $r=-0.44, P=0,01, N=32$, Fig. 2 b), pero no con la temperatura ( $r=0.19, P=0,309, N$ $=32$ ). Tampoco fue significativa la correlación entre el índice de frío y el índice de aridez $(r=0,12, P=0,500$, $N=32$ ).

Por otro lado, observamos que las plantas de la familia Arecaceae en la actualidad están presentes en climas más cálidos y secos que el encontrado en el sitio arqueológico de estudio (Fig. 3). Por lo tanto, la presencia de silicofitolitos de Arecaceae en algunas profundidades del perfil del suelo, aunque muy baja (Tabla 1), podría estar indicando que en el momento en que se generaron dichos silicofitolitos el clima fue más cálido y seco que el actual. 


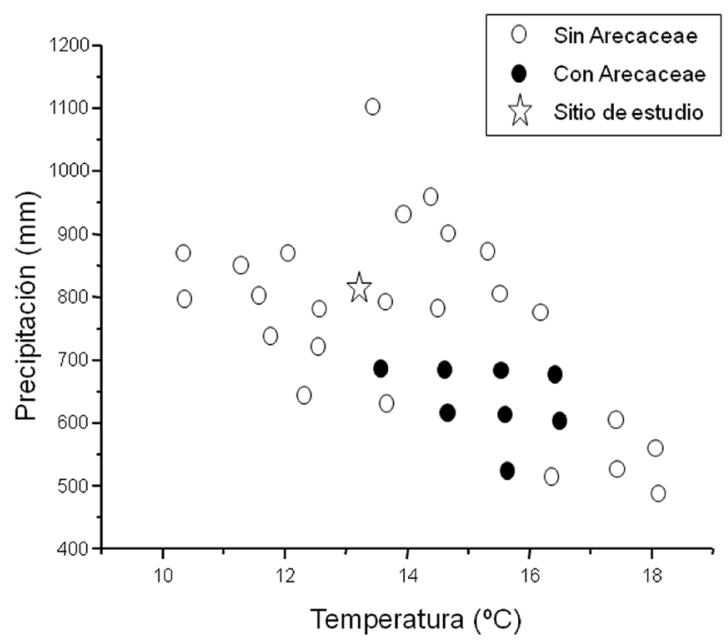

Fig. 3. Relación entre temperatura media y precipitación media de las 32 clases climáticas de las sierras de Córdoba. En negro se destacan las clases climáticas con la presencia de la familia Arecaceae. A modo de referencia, se indica también la temperatura y precipitación media del punto en el cual se encuentra el sitio arqueológico el Alto 3.

Reconstrucción paleoclimática a través de silicofitolitos

Según la reconstrucción climática a partir de los índices, hace aproximadamente diez mil años (entre los 110 y $100 \mathrm{~cm}$ de profundidad) el clima fue más frío y húmedo que el actual, pero hacia los 8000 AP $(80-90 \mathrm{~cm})$ la aridez aumentó, con poco cambio de la temperatura (Fig. 4). A partir de ese momento, la temperatura y la humedad fueron en aumento hasta llegar a un clima algo más cálido y húmedo que el actual hace aproximadamente 5000 años (entre 70 y $80 \mathrm{~cm}$ ). Luego el clima se tornó más árido y frío, hasta llegar a un clima similar al actual alrededor de los 3000 años $(60-70 \mathrm{~cm})$ (Fig. 4). Con posterioridad a esta fecha, la temperatura volvió a aumentar, pero la humedad siguió disminuyendo, por lo cual hace alrededor de 1600 años (entre los 30 y $40 \mathrm{~cm}$, aproximadamente año 400 de nuestra era) nos encontramos con un máximo de aridez y calor, que coincide además con el hallazgo de silicofitolitos de la familia Arecaceae. A partir de ese momento y hasta la actualidad, el clima se fue tornando más frío y húmedo (Fig. 4).

\section{Discusión}

Nuestros resultados aportan nuevas evidencias a favor de la aplicación del análisis de silicofitolitos como una aproximación adecuada para la reconstrucción de los cambios climáticos ocurridos durante la última etapa del Cuaternario, y avalan puntualmente su utilización en las Sierras de Córdoba.

Según nuestro conocimiento este trabajo es el primero de Argentina que utiliza datos de la distribución actual de subfamilias de gramíneas para corroborar a nivel local los índices climáticos internacionales. Encontramos una fuerte correlación entre el índice de frío construido con los datos actuales de distribución de las tres familias de Poaceae y la temperatura, como ya lo había sugerido Cabido et al. $(1997,2008)$ trabajando con la distribución de especies $\mathrm{C}_{3}$ (subfamilia Pooideae) y $\mathrm{C}_{4}$ (subfamilias Panicoideae y Chloroideae). Esto indica que el índice de frío es muy robusto y sensible a cambios en la temperatura. Por otro lado, el índice de aridez se correlacionó significativamente con la precipitación aunque la relación no fue tan alta como en el caso anterior. Por esa razón, creemos que si bien ambos índices son confiables y aplicables a esta región para reconstruir los cambios climáticos pasados de modo relativo, el índice de frío es más robusto que el índice de aridez. Por otro lado, cabe destacar que los valores absolutos de los índices calculados a partir de la vegetación actual en el sitio de estudio, fueron diferentes a los obtenidos a través de silicofitolitos para el nivel superior (entre 0 y 10 $\mathrm{cm})$. Con la distribución actual de las subfamilias se obtuvo un valor de 34,6 para el índice de frío y 21,3 para el índice de aridez, mientras que con los silicofitolitos se obtuvo un valor de 83,5 para el índice de frío y de 3 para el índice de aridez. Esto corrobora que, aunque los índices calculados con la vegetación actual son buenos indicadores climáticos, las relaciones entre estos índices y el clima actual no pueden ser utilizadas de modo directo para obtener valores absolutos de precipitación y temperatura pasadas a partir de los índices calculados con silicofitolitos. Esto posiblemente se debe a dos factores. Por un lado, la cobertura no es proporcional a la biomasa de las plantas, y por otro lado, es posible que por unidad de biomasa, distintas especies y subfamilias 


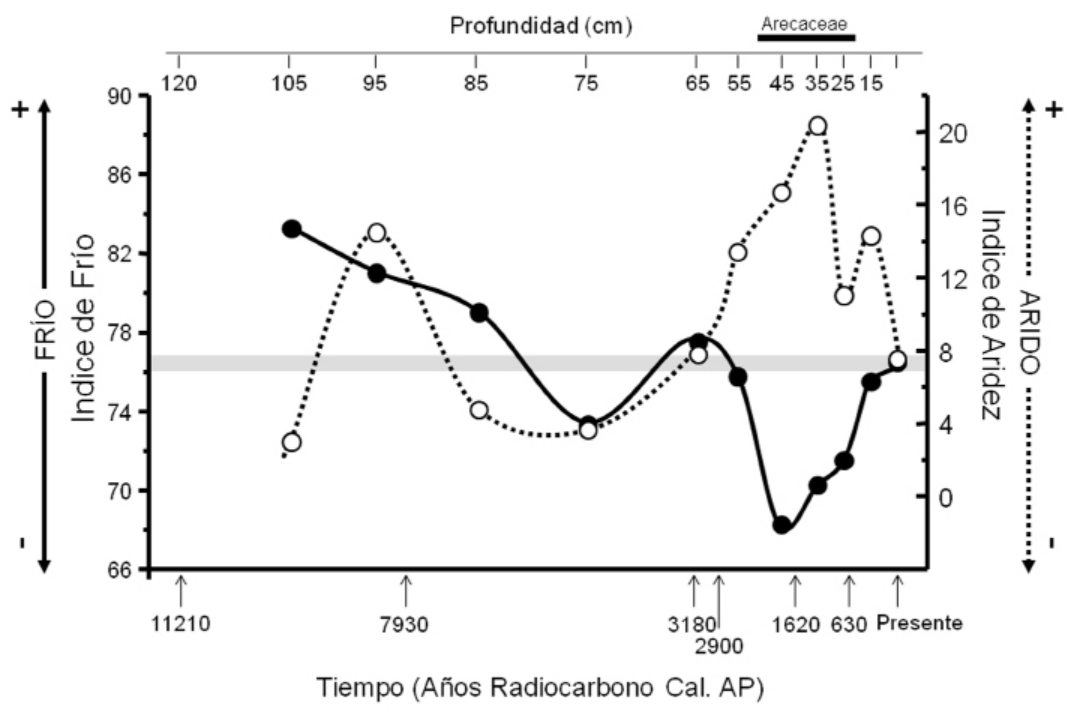

Fig. 4. índice de frío (línea negra con círculos negros) e índice de aridez (línea punteada con círculos blancos) a lo largo del tiempo (años radiocarbono calibrados antes del presente). La línea horizontal gris representa las condiciones actuales, indicadas por los valores del nivel más superficial. A los fines de una mejor visualización, utilizamos una escala lineal de tiempo con las fechas obtenidas mediante radiocarbono indicadas en el eje de las abscisas y las profundidades en la parte superior del gráfico. Para ubicar en el gráfico los puntos correspondientes a profundidades que no tenían fechado se utilizó una interpolación lineal entre pares de profundidades que sí tenían fechado.

tengan diferente producción de silicofitolitos. Sería necesario estudiar los silicofitolitos del nivel superior del suelo, producto de la vegetación actual, para intentar estimar en términos absolutos las variaciones pasadas del clima a través del análisis en el perfil del suelo.

La predominancia de silicofitolitos de la subfamilia Pooideae en todo el perfil de excavación del sitio arqueológico El Alto 3 caracteriza un clima frío a lo largo de todo el período analizado. Por otro lado, las variaciones en silicofitolitos de Chlorideaceae y Panicoideae indicaron cambios interesantes en el índice de aridez, aunque los valores absolutos siempre indican un clima relativamente húmedo. Estas características climáticas son propias del ambiente de montaña que caracteriza la provincia de Córdoba a esta altitud y en la ladera este del cordón de las Sierras Grandes (Marcora et al., 2008). Sin embargo, estas conclusiones generales a partir de los valores absolutos deben ser tomadas con cautela ya que en general se basan en descripciones de otros lados.

Las reconstrucciones climáticas de distintos autores muestran que el final del Pleistoceno y comienzos del Holoceno estuvo caracterizado por un clima más frío y árido que el actual (Vimeux et al., 2009), que desencadenó en la provincia de Córdoba grandes procesos erosivos y una marcada reducción en el nivel de agua de las Salinas Grandes, de Ambargasta y La Laguna Mar Chiquita (Carignano, 1999; Kröhling \& Carignano, 2014). Este pico de aridez puede verse representado según nuestros datos hace aproximadamente 8000 años en coincidencia con la reducción en el nivel de la laguna de Mar Chiquita observada por Piovano et al., (2009) (Fig. 5). Posteriormente a este período las reconstrucciones tanto locales como de otros lugares del mundo (Markgraf et al., 1992; Vimeux et al., 2009; Wanner et al., 2008) son consistentes al indicar que después de este último máximo glaciar caracterizado por un clima árido y frío le siguió un período con altas precipitaciones y temperaturas conocido como óptimo climático del Holoceno, que se caracterizo por un clima templado subtropical en Córdoba durante la primera parte del Holoceno (Carignano, 


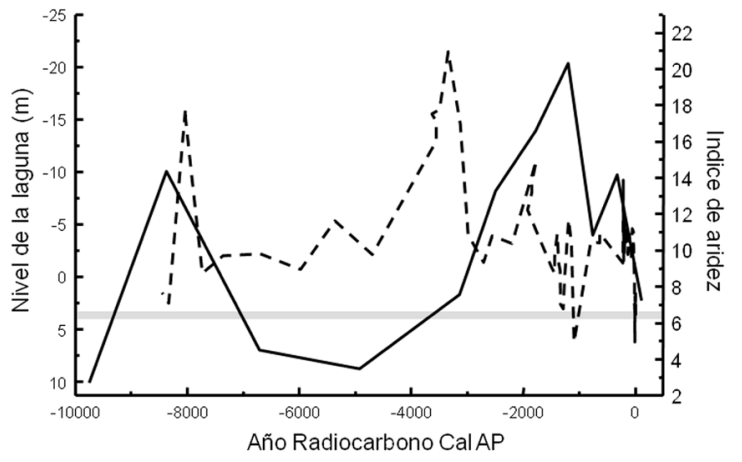

Fig 5. Cambios en el nivel de la Laguna Mar Chiquita (Córdoba) e índice de aridez en función del tiempo. La línea punteada corresponde al nivel de la laguna Mar Chiquita en relación a un nivel de base representado por el valor cero en la escala de la izquierda (representa el nivel de la laguna en 1976 antes de la gran creciente, los datos fueron adaptado de Piovano et al., 2009). Los valores más negativos (en posiciones más altas del gráfico) representan un nivel más bajo de la laguna y por lo tanto una mayor aridez. La línea sólida representa el índice de aridez del presente trabajo, y los valores más altos (en posiciones más altas del gráfico) también representan mayor aridez. La línea horizontal gris representa el presente.

1999; Piovano et al., 2009; Kröhling \& Carignano, 2014). Esta fase se observa claramente en nuestra reconstrucción, con un pico en la reducción de ambos índices hace aproximadamente 5000 años, donde coinciden valores bajos del índice de frío con valores bajos del índice de aridez, indicando un clima algo más cálido y húmedo que el actual. A partir de este momento, en la segunda mitad del Holoceno nuestros datos indican una tendencia hacia el desecamiento del clima y un aumento de la temperatura que tiene su máximo hace aproximadamente 1600 años, marcando el clima más cálido y árido del todo el período analizado. Este patrón se ve reforzado por la presencia de la familia Arecaceae característica de este tipo de condiciones climáticas, entre los años 1590 y 639 cal AP. Este período también fue registrado por Piovano et al. (2009) mostrando un pico en la desecación de la laguna Mar Chiquita en el año 3200 AP. Por otro lado Silva et al., (2011), revela un cambio de dominancia de especies
$\mathrm{C}_{4}$ a $\mathrm{C}_{3}$ alrededor de los 4.000 años antes del presente, indicando una paulatina disminución de la temperatura desde esta fecha en adelante, sugiriendo por lo tanto que el pico de calor ocurrió con anterioridad. Es decir que si bien nuestro trabajo coincide con los trabajos citados en señalar un clima más cálido y seco en el pasado reciente, hay ciertas inconsistencias en las fechas. Estas inconsistencias pueden deberse a diferencias en las técnicas utilizadas, y/o a que nuestros resultados están reducidos a un solo sitio de análisis, por lo tanto particularidades locales pueden estar explicando parte de las diferencias con los otros estudios. Por otro lado, en nuestros resultados no se observa la Anomalia climática medieval o MCA (Medieval Climatic Anomaly), caracterizada por un clima húmedo y caluroso entre 1400 y 800 AP (Cioccale, 1999; Piovano et al., 2009), lo cual puede deberse a las causas antes mencionadas. Además la reconstrucción presentada tampoco muestra la pequeña edad de hielo o LIA (Little Ice Age) caracterizada por un clima frío, que concluyó a comienzos del siglo 19 (Cioccale, 1999; Piovano et al., 2009; Kröhling \& Carignano, 2014). Sin embargo esto podría ser esperable debido a que nuestra primera datación con radio carbono es a los 630 cal AP de manera que no tenemos una resolución temporal adecuada para detectar este cambio.

Los cambios climáticos ocurridos durante el Holoceno estuvieron acompañados por grandes modificaciones en los patrones de distribución de la vegetación tanto a nivel mundial como en Sudamérica (Markgraf et al., 1992; Williams et al., 2004; Wanner et al., 2008; Vimeux et al., 2009). Sin embargo, el aumento de la población humana, junto con la extinción de la megafauna Pleistocénica, el fuego y la introducción del ganado doméstico durante los últimos miles de años, son factores clave que interactuaron con el clima acelerando o retrasando los cambios en la vegetación asociados a cambios en las condiciones climáticas (Behling et al., 2004, 2005, 2007; Mancini, 2009; Vimeux et al., 2009; Wille \& Schabitz, 2009). Estos patrones son más claros en algunos lugares de Sudamérica, sin embargo en muchos lugares como el centro de Argentina, esta información al día de hoy sigue siendo escasa (Kröhling \& Carignano, 2014), pero vital para entender los patrones de distribución presentes y futuros. 


\section{Agradecimientos}

Este trabajo fue financiado por SECYT, Universidad de Córdoba, el Inter-American Institute for Global Change Research (IAI) CRNII-2005. Los autores agradecen al IMBIV, al Consejo Nacional de Investigaciones Científicas y Técnicas (CONICET) y a la Universidad Nacional de Córdoba por las facilidades brindadas para llevar a cabo esta investigación. Y finalmente a dos revisores anónimos por sus valiosos comentarios y sugerencias. MG, ML, DG y AC, son investigadores de CONICET y MG, DG y AC docentes de la UNC.

\section{Bibliografía}

ANDREIS, R. R. 1972. Paleosuelos de la Formación Musters (Eoceno Medio), Laguna del Mate, Prov. de Chubut. Rep. Argentina. Revista Asociación Argentina de Mineralogia, Petrología $y$ Sedimentología 3: 91-97.

BEHLING, H. \& V. PILLAR. 2007. Late Quaternary vegetation, biodiversity and fire dynamics on the southern Brazilian highland and their implication for conservation and management of modern Araucaria forest and grassland ecosystems. Phil. Trans. Roy. Soc. B: Biol. Sci. 362: 243.

BEHLING, H., V. PILLAR \& S. BAUERMANN. 2005. Late Quaternarygrassland (Campos), gallery forest, fire and climate dynamics, studied by pollen, charcoal and multivariate analysis of the Sao Francisco de Assis core in western RioGrande do Sul (southern Brazil). Rev. of Palaeobotany and Palynology 133: 235-248.

BEHLING, H., V. PILlAR, L. ORLOCI \& S. BAUERMANN. 2004. Late Quaternary Araucaria forest, grassland (Campos), fire and climate dynamics, studied by high-resolution pollen, charcoal and multivariate analysis of the Cambar. do Sul core in southern Brazil. Palaeogeography, Palaeoclimatology, Palaeoecology 203: 277-297.

BERTOLDIDEPOMAR, H. 1971. Ensayo de clasificación morfológica de los silicofitolitos. Ameghiniana 8: 317-328.

BERTOLDI DE POMAR, H. 1975. Los silicofitolitos: Sinopsis de su conocimiento. Darwiniana 19: 173206.

BONOMO, M., A.F. ZUCOL, B. GUTIÉRREZ TÉLLEZ, A. CORADEGHINI \& M.S. VIGNA. 2009. Late Holocene palaeoenvironments of the Nutria Mansa 1 archaeological site, Argentina. J. Paleolimnol. 41: 273-296

BORRELLI, N., M. OSTERRIETH \& J.
MARCOVECCHIO. 2008. Interrelations of vegetal cover, silicophytolith content and pedogenesisof Typical Argiudolls of the Pampean Plain, Argentina. Catena 75: 146-153

BORRELLI, N., M. FERNÁNDEZ HONAINE, S. M. ALTAMIRANO \& M. OSTERRIETH. 2011. Calcium and silica biomineralizations in species associated to aquatic environments of the Pampean Plain, Argentina. Aquatic Bot. 94: 29-36.

BOYD, M. 2005. Phytoliths as paleoenvironmental indicators in a dune field on the northern Great Plains. J. Arid Environ. 61: 357-375.

BREMOND, L., A. ALEXANDRE, M. J. WOOLLER, C. HÉLY, D.WILLIAMSON, P. A. SCHÄFER, A. MAJUL, \& J. GUIOT. 2008. Phytolith indices as proxies of grass subfamilies on East African tropical mountains. Global Planet. Change 61: 209-224.

CABIDO, M., N. ATECA, M. ASTEGIANO \& A.M. ANTON. 1997. Distribution of C3 and C4 grasses along an altitudinal gradient in Central Argentina. $J$. Biogeogr. 24: 197-204.

CABIDO, M., E. PONS, J. CANTERO, J. LEWIS \& A. ANTON. 2008. Photosynthetic pathway variation among $\mathrm{C} 4$ grasses along a precipitation gradient in Argentina. J. Biogeogr. 35: 131-140.

CAPITANELLI, J. 1979. Geomorfología. In: VAZQUEZ, J., R. MIATELLO \& M. ROQUE (eds.), Geografía Física de la Provincia de Córdoba, pp. 213-296. Editorial Boldt, Buenos Aires.

CARIGNANO, C. A. 1999. Late Pleistocene to recent climate change in Córdoba Province, Argentina: Geomorphological evidence. Quatern. Int. 57/58: 117-134.

CINGOLANI, A. M., M. V. VAIERETTI, D. E. GURVICH, M. A. GIORGIS \& M. CABIDO. 2010. Predicting alpha, beta and gamma plant diversity from physiognomic and physical indicators as a tool for ecosystem monitoring. Biol. Conserv. 143: 2570-2577.

CINGOLANI, A.M., I. NOY-MEIR, D. RENISON \& M. CABIDO. 2008. La ganadería extensiva, ¿es compatible con la conservación de la biodiversidad y los suelos? Ecología Austral 18: 253-271.

CIOCCALE, M. 1999. Climatic fluctuations in the Central Region of Argentina in the last 1000 years. Quatern. Int. 62: 35-47.

DE FINA, A. L. 1992. Aptitud Agroclimática de la República Argentina. Academia Nacional de Agronomía y Veterinaria, Buenos Aires, p. 402.

DEL PUERTO, L., F. GARCÍA-RODRÍGUEZ, H. INDA, R. BRACCO, C. CASTIÑEIRA \& J. B. ADAMS. 2006. Paleolimnological evidence of Holocene climatic changes in Lake Blanca, southern Uruguay. J. Paleolimnol. 36: 151-163.

FERNÁNDEZ HONAINE, M., A. ZUCOL \& M. 
OSTERRIETH. 2006. Phytolith assemblage and systematic association in grassland species of the SE Pampean Plains, Argentina. Ann. Bot. 98: 1155-1165.

FERNÁNDEZ HONAINE, M., M.OSTERRIETH \& A. ZUCOL. 2009a. Plant communities and soil phytolith assemblages relationship in native grasslands from southeastern Buenos Aires Province, Argentina. Catena 76: 89-96.

FERNÁNDEZ HONAINE, M., A. ZUCOL \& M. OSTERRIETH. 2009b. Phytolith analysis of Cyperaceae from the Pampean Region, Argentina. Aust. J. Bot. 57: 512-523.

FREDLUND, G. G. \& L. T. TIESZEN. 1994. Modern phytolith assemblages from the North American Great Plains. J. Biogeogr. 21: 312-335.

GAlleGO, L., \& R. A. DISTEL. 2004. Phytolith assemblages in grasses native to Central Argentina. Ann. Bot. 94: 865-874.

GALlEGO, L., R. A. DISTEL, R. CAMINA \& R. M. RODRIGUEZ IGLESIAS. 2004. Soil phytoliths as evidence for species replacement in grazed rangelands of Central Argentina. Ecography 27: 1-8.

GIORGIS, M. A., A. M. CINGOLANI, F. CHIARINI, J. CHIAPELLA, G. BARBOZA, L. ARIZA ESPINAR, R. MORERO, D. E. GURVICH, P. A. TECCO, R. SUBILIS \& M. CABIDO. 2011. Composición florística del Bosque Chaqueño Serrano de la provincia de Córdoba, Argentina. Kurtziana 36: 9-43.

HIJMANS, R. J., S. E. CAMERON, J. L. PARRA, P. G. JONES \& A. JARVIS. 2005. Very high resolution interpolated climate surface for global land areas. Int. J. Climat. 25: 1965-1978.

HOYOS, L. 2012. Factores relacionados a la deforestación en bosques del chaco seco de la Provincia de Córdoba. Universidad Nacional de Córdoba, Facultad de Ciencias Exactas, Físicas y Naturales. Tesis doctoral inédita.

KORSTANJE, M. A. \& P. CUENYA. 2008. Arqueología de la Agricultura: Suelos y Microfósiles en campos de cultivo del valle del Bolsón, Catamarca, Argentina. En M. A. KORSTANJE \& M. DEL P. BABOT (eds.), Matices Interdisciplinarios en Estudios Fitolíticos y de Otros Microfósiles. pp. 133-148. BAR International Series S1870.

KORSTANJE, M. A. \& P. CUENYA. 2010. Ancient agricultura and domestic activities: a contextual approach studying silica phytoliths and other microfossils in soil. Environ. Archaeology 15:43-63.

KORSTANJE, M. A. 2005. La Organización del Trabajo en torno a la Producción de Alimentos en Sociedades Agropastoriles Formativas (Provincia de Catamarca, República Argentina). Tesis doctoral en Arqueología. Instituto de Arqueología y Museo. Facultad de Ciencias Naturales e I.M.L. Universidad Nacional de Tucumán. Tucumán. Inédita.
KORStanje, M. A. 2009. Microfósiles y agricultura prehispánica: primeros resultados de un análisis múltiple en el N.O.A. En: ZUCOL, A.F. , M. OSTERRIETH \& M. BREA (eds.), Fitolitos: Estado Actual de su Conocimiento en América del Sur, pp. 249-263. Mar del Plata: Universidad de Mar del Plata, Argentina.

KRÖHLING, D. \& C. A. CARIGNANO 2014. La estratigrafía de los depósitos sedimentarios cuaternarios. En: MARTINO, R. D. \& A. B. GUERESCHI (eds), Relatorio del XIX Congreso geológico Argentino: geología y recursos naturales de la provincia de Córdoba, pp. 673724. Alsapema S.A. Córdoba. Argentina.

LOMOLINO, M. V., B. R. RIDDLE, R. J. WHITTAKER \& J. H. BROWN. 2010. Biogeography, 4th Edition. Sinauer Associates.

LÓPEZ, M. L. 2007. Los vegetales comestibles en la economía prehispánica tardía de Sierras Centrales. Estudios arqueobotánicos en el Valle de Punilla y el Valle de Salsacate (Provincia de Córdoba). Tesis de Licenciatura. Escuela de Historia, Facultad de Filosofía y Humanidades. Universidad Nacional de Córdoba.

MADELLA, M., A. ALEXANDRE \& T. BALL. 2005. International Code for Phytolith Nomenclature 1.0. Ann. Bot. 96: 253-260.

MANCINI, M. 2009. Holocene vegetation and climate changes from a peat pollen record of the forest-steppe ecotone, Southwest of Patagonia (Argentina). Quaternary Sci. Rev. 28: 1490-1497.

MARCORA, P., I. HENSEN, D. RENISON, P. SELTMANN \& K. WESCHE. 2008. The performance of Polylepis australis trees along their entire altitudinal range: implications of climate change for their conservation. Divers. Distrib. 14: 630-636.

MARKGRAF, V., J. R. DODSON, A. P. KERSHAW, M. S. MCGLONE \& N. NICHOLLS. 1992. Evolution of late Pleistocene and Holocene climates in the circum-South Pacific land areas. Clim. Dynam. 6: 193-211.

MEDINA, M. E., M. L. LÓPEZ \& E. E. BERBERIÁN. 2009. Agricultura y recolección en el tardío prehispánico de las Sierras de Córdoba (Argentina): el registro arqueobotánico de C. Pun. 39. Arqueología 15: 217-230.

MOONEY, H. A. \& J. R. EHLERINGER. 1997. Photosynthesis. In: CRAWLEY M. (ed.), Plant Ecology, pp. 1-27. Blackwell Science, Oxford.

OSTERRIETH, M., M. MADELLA, D. ZURRO \& M. FERNANDEZ-ALVAREZ. 2009. Taphonomical Aspects of Silica Phytoliths in the Loess Sediments of the Argentinean Pampas. Quat. Int. 193: 70-79. 
OSTERRIETH, M. 2006. Silicofitolitos en suelos, paleosuelos y sedimentos. Actas III Congreso Argentino de Cuaternario y Geomorfología, FCEFyN, Córdoba, pp. 351-365.

OSTERRIETH, M., G. MARTÍNEZ, D. ZURRO, A. ZUCOL, M. BREA \& D. MAZZANTI. 2002. Procesos de formación del Sitio 2 de la localidad arqueológica Amalia: evolución paleoambiental. In: MAZZANTI, D., M. BERÓN \& F. OLIVA (eds.), Del Mar a los salitrales. Diez mil años de historia pampeana en el umbral del tercer milenio. pp. 343-354. Facultad de Humanidades, UNMdP, Mar del Plata.

OSTERRIETH, M; G. MARTINEZ; M. GUTIERREZ \& F. ALVAREZ. 2008. Biomorfos de sílice en secuencias pedoarqueológicas del sitio Paso Otero 5, Buenos Aires. British Archaelogical Research: 77-90.

PEARSALL, D. M. 1989. Paleoethnobotany. A Handbook of Procedures. San Diego: Academic Press. 470 pp.

PIOVANO, E. L., D. ARIZTEGUI, F. CÓRDOBA, M. CIOCCALE \& F. SYLVESTRE. 2009. Hydrological Variability in South America Below the Tropic of Capricorn (Pampas and Patagonia, Argentina) During the Last 13.0 Ka. In: VIMEUX F., F. SYLVESTRE \& M. KHODRI (eds.), Past Climate Variability in South America and Surrounding Regions, pp. 323-351. Springer, France.

PIPERNO, D. R. 2006. Phytoliths: A Comprehensive Guide for Archaeologists and Paleoecologists. Altamira. Oxford, UK.

RIVERO, D. \& F. ROLDÁN. 2005 Inicial peopling of the Córdoba Mountains, Argentina: first evidence from El Alto 3. Current Research in The Pleistocene 22: 33-35.

RIVERO, D. \& L. LÓPEZ. 2011. Evidencias del procesamiento de recursos vegetales por cazadoresrecolectores de las Sierras de Córdoba en el período ca. 7000-2900 AP. Resúmenes de las IX Jornadas de Investigadores en Arqueología y Etnohistoria del Centro-Oeste del país, Universidad Nacional de Río Cuarto, Río Cuarto: 49-50.

RIVERO, D. \& M.L. LÓPEZ. 2010. Procesamiento de vegetales durante el Holoceno Temprano en las sierras de Córdoba (ca. 7100 AP). Primera aproximación". En: BÁRCENA J.R. \& H. CHIAVAZZA (eds.), Arqueología Argentina en el Bicentenario de la Revolución de Mayo. Actas del XVII Congreso Nacional de Arqueología Argentina. Mendoza, 11-15 de Octubre del 2010. Tomo V, Capítulo 33, pp. 17471752.

RIVERO, D. 2007 "Los primeros pobladores de las Sierras Centrales de Argentina. Las evidencias más antiguas del sitio "El Alto 3" (Dpto. Punilla, pcia. de Córdoba)". Comechingonia Virtual. Revista Electrónica de Arqueología 1:79-123.
RIVERO, D. 2009. Ecología de cazadores-recolectores del sector central de las Sierras de Córdoba (Rep. Argentina). BAR International Series 2007, Oxford.

ROVNER, I. 1971. Potential of opal phytoliths for use in paleoecological reconstruction. Quat. Res. 1: 343-359.

SANABRIA, J., G. ARGÜELLO, D. RIVERO \& E. BERBERIÁN. 2009. cambios climáticos durante el Pleistoceno Tardío- Holoceno en la localidad arqueológica "El Alto 3". Sierra grande de Córdoba. Argentina. J. SANABRIA, G. ARGÜELLO, D. RIVERO Y E. BERBERIÁN. EN J. SAYAGO Y M. COLLANTES (eds.), Geomorfología y cambio climático, pp. 137-144, Editado por la Universidad Nacional de Tucumán.

SILVA, L.C.R., M. A., GIORGIS, A. MADHUR, L. ENRICO, N. PÉREZ-HARGUINDEGUY, V. FALCZUK, L. TIESZEN \& M. CABIDO. 2011. Evidence of shift in $\mathrm{C} 4$ species range in central Argentina during the late Holocene. Plant and Soil. 349: 261-27.

SORENG, R.J., G. DAVIDSE, P.M. PETERSON, F. O. ZULOAGA, E. J. JUDZIEWICZ,T. S. FILGUEIRAS \& O. MORRONE. 2012. A world-wide phylogenetic classification of Poaceae (Gramineae). Catalogue of New World Grasses.

SORENG, R.J., G. DAVIDSE, P.M. PETERSON, F. O. ZULOAGA, E. J. JUDZIEWICZ,T. S. FILGUEIRAS \& O. MORRONE. 2015. A worldwide phylogenetic classification of the Poaceae (Gramineae). J. Syst. Evol. 53: 117-137.

STRASSER, E., J. CHIESA, M. OSTERRIETH, E. MARCHEVSKY \& E. PERINO. 2006. Phytoliths as palaeonvironmental indicators. XXIV Reunión Científica Anual de la Sociedad de Biología de Cuyo - IV Reunión Científica de la Sociedad Argentina de Microscopía (SAMIC), San Luis (Argentina), p. 160.

TWISS, P.C. 1992. Predicted world distribution of C3 and C4 grass phytolith. In: Rapp G., S. C. Mulholland (eds.), Phytolith systematics. Emerging issues. Advances in Archaeological and Museum Science, pp. 113-128 Plenum Press, New York.

TWISS, P.C., E. SUESS \& R. SMITH. 1969. Morphological classification of grass phytoliths. Proc. Soil Sci. Soc. Am. 33: 109-115.

VIMEUX, F., F. SYLVESTRE \& M. KHODRI. 2009. Past climate variability in South America and surrounding regions: from the Last Glacial Maximum to the Holocene. Springer Verlag.

WANNER, H., J BEER, J. BÜTIKOFER, T. CROWLEY, U. CUBASCH, J. FLÜCKIGER, H. GOOSSE, M. GROSJEAN, F. JOOS \& J. KAPLAN. 2008. Mid-to Late Holocene climate change: an overview. Quaternary Sci. Rev. 27: 1791-1828.

WENINGER, B., O. JÖRIS \& U DANZEGLOCKE. 2007 CALPAL. Cologne Radiocarbon Calibration 
\& Paleoclimate Research Package. Disponible en: www.calpal.de

WILLE, M. \& F. SCHABITZ. 2009. Late-glacial and Holocene climatedynamics at the steppe/forest ecotone in southernmost Patagonia, Argentina: the pollen record from a fen near Brazo Sur, Lago Argentino. Vegetation History and Archaeobotany 18: 225-234.

WILliAMS, J., B. SHUMAN, T. III. WEBB, P. BARTLEIN \& P. LEDUC. 2004. Late-Quaternary vegetation dynamics in North America: scaling from taxa to biomes. Ecol. Monogr. 74: 309-334.

ZUCOL, A. F. \& M. BREA. 2000. Análisis fitolítico de la Formación Paraná (Mioceno superior) en el departamento Diamante, Entre Ríos, Argentina. Libro de resúmenes del II Congreso Latinoamericano de sedimentología y VIII Reunión Argentina de Sedimentología, p. 190. Mar del Plata.

ZUCOL, A. F. \& M. BREA. 2001. Asociación fitolítica de la Formación Alvear (Pleistoceno Inferior), Entre Ríos, Argentina. Ameghiniana 38 (4-suplemento): 49R. Buenos Aires.

ZUCOL, A. F. 1996. Microfitolitos de las Poaceae argentinas: I. Microfitolitos foliares de algunas especies del género Stipa (Stipeae: Arundinoideae), de la Provincia de Entre Ríos. Darwiniana, 34: 151172.

ZUCOL, A. F. 2001. Fitolitos III. Una nueva metodología descriptiva. Asociaciones fitolíticas de Piptochaetium montevidense (Stipeae: Poaceae). Bol. Soc. Arg. Bot. 36: 69-85.

ZUCOL, A. F., M. BREA, A. LUTZ \& L. M. ANZOTEGUI. 2004. Aportes al conocimiento de la paleobiodiversidad del Cenozoico superior del Litoral argentino: Estudios Paleflorísticos INSUGEO, Miscelánea 12: 91 - 102.

ZUCOL, A.F. 1998. Microfitolitos de las Poaceae argentinas: II. microfitolitos foliares de algunas especies del género Panicum (Poaceae, Paniceae) de la provincia de Entre Ríos. Darwiniana 36: 29-50.
ZUCOL, A.F. 1999. Fitolitos de las Poaceae Argentinas: IV. Asociación fitolítica de Cortaderia selloana (Danthonieae: Poaceae), de la Provincia de Entre Ríos (Argentina). Natura Neotropicalis 30: 25-33.

ZUCOL, A.F. 2000. Fitolitos de Poaceae en Argentina. III. Fitolitos foliares de especies del género Paspalum (Paniceae) en la provincia de Entre Ríos. Darwiniana 38: 11-32.

ZUCOL, A.F., B. GUTIERREZ TELLEZ \& M. BONOMO M. 2003 Estudios etnobotánicos del sitio arqueológico Nutria Mansa 1 (Partido de Gral. Alvarado, provincia de Buenos Aires): I. Análisis de fitolíticos y diatomológicos. Resúmenes del XII Simposio Argentino de Paleobotánica y Palinología. Museo Argentino de Ciencias Naturales, Buenos Aires, p 58.

ZUCOL, A. F., M. BREA \& E. PASSEGGI. 2008. Los estudios fitolíticos en América del Sur, una visión retrospectiva. En: KORSTANJE M. A. \& M. DEL P. BABOT (eds.), Matices Interdisciplinarios en Estudios Fitolíticos y de Otros Microfósiles, pp. 3-21. BAR International Series S1870.

ZUCOL, A. F., M. BREA, A. A. CARLINI \& R.H. MADDEN. 2001. Análisis fitolíticos en la secuencia sedimentaria de Gran Barranca, Chubut, Argentina: II. El Miembro Colhué Huapi (Formación Sarmiento). Ameghiniana 38 (4-suplemento): 50R. Buenos Aires.

ZUCOL, A.F., M. BREA, M. OSTERRIETH \& G. MARTÍNEZ. 2002. Análisis fitolítico de un horizonte sedimentario del Sitio 2 de la localidad arqueológica Amalia (Holoceno temprano). In: MAZZANTI, D., M. BERÓN \& F. OLIVA (eds.), Del Mar a los salitrales. Diez mil años de historia pampeana en el umbral del tercer milenio, pp. 355-363. Facultad de Humanidades, UNMdP, Mar del Plata.

Recibido el 18 de diciembre de 2014, aceptado el 5 de mayo de 2015. 
Apéndice 1. Principales sílicofitolitos analizados. A: Bilobados halteriformes (a-h bilobados simples; i-j tipo Stipa). B: Polilobados halteriformes. C: Mitad-bilobado halteriformes. D: Circulares/ovalados. E: Prismáticos cuadrados y rectangulares. F: Cuneiformes. G: Estróbilo de base cóncava y ápice sin ornamentación. H: Silla de montar. I: Globulares de superficie espinosa. Barras de escala $=10 \mu \mathrm{m}$.

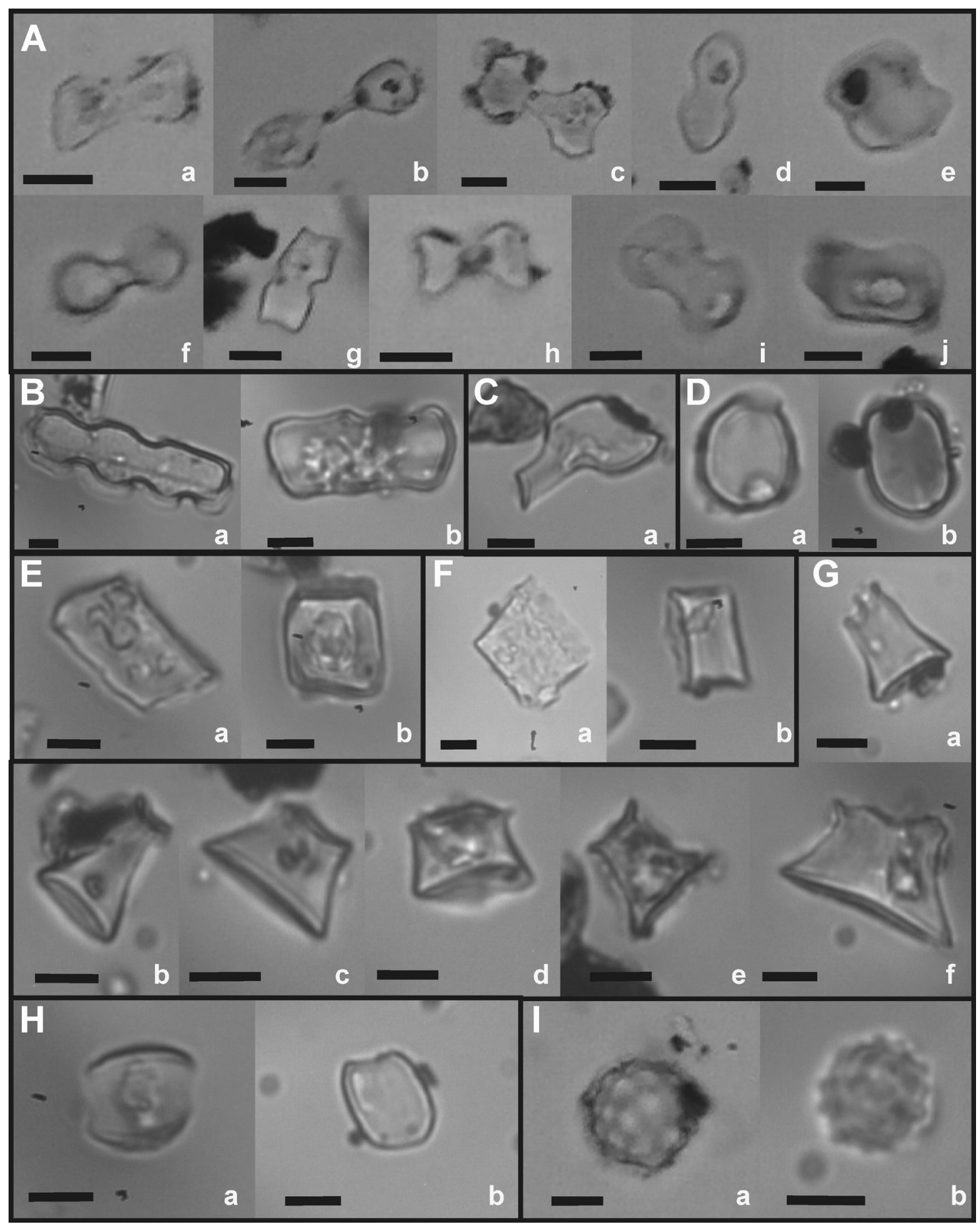


